\title{
Complementarity in the Bohr-Einstein Photon Box
}

\author{
Dennis Dieks and Sander Lam \\ History and Foundations of Science \\ Utrecht University, P.O. Box 80.000 \\ 3508 TA Utrecht, The Netherlands
}

\begin{abstract}
The photon box thought experiment can be considered a forerunner of the EPR-experiment: by performing suitable measurements on the box it is possible to "prepare" the photon, long after it has escaped, in either of two complementary states. Consistency requires that the corresponding box measurements be complementary as well. At first sight it seems, however, that these measurements can be jointly performed with arbitrary precision: they pertain to different systems (the center of mass of the box and an internal clock, respectively). But this is deceptive. As we show by explicit calculation, although the relevant quantities are simultaneously measurable, they develop non-vanishing commutators when calculated back to the time of escape of the photon. This justifies Bohr's qualitative arguments in a precise way; and it illustrates how the details of the dynamics conspire to guarantee the requirements of complementarity. In addition, our calculations exhibit a "fine structure" in the distribution of the uncertainties over the complementary quantities: depending on when the box measurement is performed, the resulting quantum description of the photon differs. This brings us close to the argumentation of the later EPR thought experiment.
\end{abstract}

\section{Introduction}

The 1930 Solvay conference was the scene of the famous weighing-of-energy debate between Einstein and Bohr. According to Bohr's report [1], the debate revolved around the validity of the time-energy uncertainty relation. As Bohr tells us, Einstein had devised an ingenious thought experiment (involving a "photon box") with which he wanted to demonstrate that an individual photon can have both a sharply defined energy and a precisely predictable time of arrival at a detector. If successful, this would mean the demise of the time-uncertainty relation. Einstein himself later maintained that Bohr had misunderstood his 
intentions: that it was not the validity of the uncertainty relation, but rather the unpalatable implications of complementarity in the case of correlated distant systems that he was targeting. Considered either way, the thought experiment furnishes a remarkable illustration of quantum mechanical complementarity.

The idea of the experiment is to start with a box, filled with radiation, hanging stationary in the gravitational field (after a preliminary balancing procedure). In this situation the total energy of the box and its contents has a well-defined value ${ }^{1}$. Inside of the box there is a clock that opens a small shutter when its hands reach a fixed position. This shutter remains open for a very brief time interval, during which one photon escapes. After the photon's escape (possibly at a much later time), the box is weighed, so that its mass - and therefore its energy - can be determined. Comparison with the initial situation gives us the energy of the escaped photon. In addition we can read off the internal clock, and this will tell us how much time has elapsed since the opening of the shutter.

The weighing is performed by looking at how the center of mass of the box has moved under the influence of gravity since the photon's escape ${ }^{2}$. Since the dynamical quantities of the center of mass commute with the variables of the internal clock, it is possible to perform a measurement in which both the position of the hands of the clock and the position (or momentum) of the center of mass of the box are sharply determined. It therefore appears clear that both the energy and the time of escape of the photon can be determined with arbitrary precision. That, however, would imply a violation of the time-energy uncertainty principle applied to the photon - quantum mechanics must in some way forbid this joint precise determination. Consistency requires that the mass of the box and the opening time of the shutter, as determined from the weighingplus-reading-off-the-clock measurement, be complementary quantities. To show exactly how quantum mechanics makes this happen is the main purpose of this note.

Bohr writes that some time after the original debate

"[Ehrenfest] told me that Einstein was far from satisfied and with his usual acuteness had discerned new aspects of the situation which strengthened his critical attitude. In fact, by further examining the possibilities for the application of a balance arrangement, Einstein had perceived alternative procedures which, even if they did not allow the use he originally intended, might seem to enhance the paradoxes beyond the possibilities of logical solution. Thus, Einstein had pointed out that, after a preliminary weighing of the box with

\footnotetext{
${ }^{1}$ Since the box system has a very large mass, the spread in energy can be vanishingly small even though the spreads in position and momentum of its center of mass do not vanish.

${ }^{2}$ In fact, Bohr suggests to bring the box back to its zero position by attaching suitable loads. This is just another way of studying the center of mass quantities. Therefore, although our calculations are based on a slightly different weighing procedure, the underlying principle applies equally to the procedure that was analysed by Bohr.
} 
the clock and the subsequent escape of the photon, one was still left with the choice of either repeating the weighing or opening the box and comparing the reading of the clock with the standard time scale. Consequently, we are at this stage still free to choose whether we want to draw conclusions either about the energy of the photon or about the moment when it left the box." ([1], pp. 228-229).

Thus, according to this account Einstein later shifted towards EPR-like considerations: apparently having been convinced at the Solvay conference by Bohr's arguments, Einstein now started pointing out that even if the photon is already far away, we can still decide to perform one or the other of a pair of complementary measurements and thus "prepare" the photon in either a state with a well-defined energy or in a state peaked in time. This is highly remarkable, since we would expect the distant photon to be a system on its own, with properties that cannot depend on what happens outside of its lightcone. As already mentioned, it may be that Bohr misinterpreted the logic of the sequence of events here: perhaps Einstein already accepted the validity of the uncertainty relations in 1930 and meant his photon box experiment from the start as a kind of delayed choice experiment. This is in fact what is suggested by looking at the precise text of Ehrenfest's message to Bohr. Ehrenfest visited Einstein in 1931 and found him to be very outspoken about the purpose of the photon box. In his letter to Bohr of 9 July 1931, Ehrenfest reported: "He said to me that, for a long time already, he absolutely no longer doubted the uncertainty relations, and that he thus, e.g., had BY NO MEANS invented the "weighable light-flash box' 'contra uncertainty relation', but for a totally different purpose" [5].

Regardless of whether Bohr's or Einstein's account is closer to the truth, it is an important point that the box-plus-photon system furnishes an example of simultaneously existing strict correlations between complementary quantities. Einstein's thought experiment can be considered a forerunner of the EPRexperiment. These general features of the experiment we shall address first (see also [3] for references to earlier discussions of the photon box).

\section{Global Analysis of the Experiment}

An essential element of the photon-box experiment is the correlation that exists between box and photon quantities after the photon has escaped. The relevant photon quantities are its energy $E_{p h}$ and its time of arrival at a given detector, $T_{a r r}$. These are correlated with the box energy $E$ and the position of the hands of the clock in the box, $q_{c l}$, respectively. The two energies are correlated as a consequence of total energy conservation; and since the clock allowed the photon to escape when its hands were at a fixed predetermined position, $q_{c l}=0$, say, after which the photon travelled with the fixed speed $c, q_{c l}$ and $T_{a r r}$ are also correlated. 
The two photon quantities, energy and time of arrival, are complementary according to quantum mechanics. Indeed, an energy eigenstate is a plane wave, which obviously does not have a well-defined time of arrival at a given point; conversely a very narrow wave packet contains very many different frequencies, and therefore is a superposition of different energy states. Paradoxically, it nevertheless seems that these two photon quantities can simultaneously be determined, with arbitrary precision, via judicious measurements on the box.

But if quantum mechanics is consistent, any uncertainty relation valid for the photon quantities must obviously have its counterpart in an uncertainty relation for the correlated box quantities - it should be impossible to beat the uncertainty relation for photon quantities by measuring correlated box quantities. We should therefore expect that the box cannot possess both a definite value of its energy and a definite time at which the shutter opened. In view of the correlations between the box and the photon, the total state of these two systems has to be entangled, such that neither the energies nor the relevant time quantities of both box and photon are sharply defined, whereas their correlation is a sharply defined quantity of the total system ${ }^{3}$.

According to relativity, all energy possesses mass and is acted upon by gravity. The experiment starts with the box in a stationary position after a preliminary weighing, but after the escape of the photon the box experiences a net force, which depends on the mass of the escaped photon, $m$. As a consequence, the box starts moving and both the position $q$ and the momentum $p$ of its center of mass get correlated to $m$. A measurement of either $p$ or $q$ will therefore provide information about $m$. On the other hand, reading off the time indicated by the clock, $q_{c l}$, will yield information about how much time has passed since the shutter was opened. Both $q_{c l}$ and either $p$ or $q$ can be measured and can jointly have sharp values, since these quantities pertain to different systems - the internal clock and the center of mass of the box, respectively - and commute.

We have already argued that the escape time calculated from $q_{c l}$, and on the other hand the box energy as computed from a measurement of either $p$ or $q$, must be complementary quantities. This should be reflected by a non-vanishing value of the commutator of the operators representing these quantities. That this is indeed so, and that this leads to exactly the right uncertainty relations, is what we shall show now.

\footnotetext{
${ }^{3}$ This in turn means that the box and the photon will not have their own pure states: both must be described by mixed states. These mixed states can be written as mixtures of well-defined mass states, or alternatively as mixtures of states peaked in time. As long as we discuss the two systems separately, we may think of these mixtures as classical "ignorance mixtures" without getting into contradictions.
} 


\section{Complementarity of Mass and Escape Time}

We start our considerations with the joint measurement of either $p$ or $q$ of the center of mass of the box, together with the clock variable $q_{c l}$. This measurement takes place when the photon is already well on its way. The chosen center of mass quantity and the clock variable may both be measured with arbitrary precision: the corresponding operators commute. However, in order to be able to say something about the photon, we have to calculate back to values of the relevant quantities at the time the shutter opened and the photon escaped. To make this stand out in the calculations below, we choose the origin of time, $t=0$, at the moment of the final measurement and choose the positive time direction backwards, i.e. going into the direction of the earlier photon emission event. We shall show that the state of the box corresponding to the measurement result (this state can be thought of as resulting from application of the projection postulate, or "collapse of the wavefunction" to the pre-measurement box state), when followed back in time to the instant of the photon emission, exhibits quantum spreads that exactly lead to the expected - and requireduncertainties in $E_{p h}$ and $T_{a r r}$.

Taking into account that during the motion of the box the clock finds itself at different heights in the gravitational field, depending on the position of the center of mass, $q$, we have the following expression for the clock variable at time $t$ before the final measurement $(t=0$ corresponds to the final measurement and $t$ is counted backwards):

$$
q_{c l}(t)=\int_{0}^{t} d \tau\left(1-\frac{g q(\tau)}{c^{2}}\right) .
$$

Bohr took recourse to general relativity to justify the use of this formula, but since then it has be shown [7] that Eq. (1) follows from just the assumption that energy has mass and can be weighed (which is exactly what Einstein needed to posit in order to make the thought experiment work in the first place). This is an important point that makes the analysis of the experiment self-contained: it would not be satisfactory if only by invoking general relativity the consistency of quantum mechanics could be demonstrated.

From (1) we see that

$$
\dot{q}_{c l}(t)=1-\frac{g}{c^{2}} q(t) .
$$

For the Heisenberg equations of motion of the vertical position and momentum of the box we have, with the same convention about $t$ :

$$
\dot{q}(t)=p / M \quad, \quad \dot{p}(t)=-m g-V^{\prime}(q),
$$

where $V(q)$ is the potential in which the box finds itself (in Bohr's description of the experiment the box is suspended from a spring, which would correspond to 
$V(q)=1 / 2 k q^{2}$, with $k$ the spring constant); the prime indicates differentiation with respect to vertical position.

The commutators $\left[p, q_{c l}\right]$ and $\left[q, q_{c l}\right]$ both vanish at $t=0$; this means that in the measurement sharp values can be assigned to both $q_{c l}$ and either $p$ or $q$. But in the Heisenberg picture the commutators change in time and do not remain null. For the change of the commutator of $p$ and $q_{c l}$ we can write down the following differential equation:

$$
\frac{d}{d t}\left[p, q_{c l}\right]=\left[\dot{p}, q_{c l}\right]+\left[p, \dot{q}_{c l}\right]=\frac{g}{c^{2}} i \hbar-\left[V^{\prime}(q), q_{c l}\right]
$$

where (2) and (3) have been used. Similarly, we find for the commutator between $q$ and $q_{c l}$ :

$$
\frac{d}{d t}\left[q, q_{c l}\right]=\left[\dot{q}, q_{c l}\right]+\left[q, \dot{q}_{c l}\right]=\left[\dot{q}, q_{c l}\right]=\frac{1}{M}\left[p, q_{c l}\right] .
$$

It follows that in the simplest situation, in which $V^{\prime}$ vanishes and the box only experiences the force of gravity, we have

$$
\left[p, q_{c l}\right]=\frac{g}{c^{2}} i \hbar t
$$

and

$$
\left[q, q_{c l}\right]=\frac{g}{2 M c^{2}} i \hbar t^{2} .
$$

So the center of mass coordinates develop (recall: backwards in time!) nonvanishing commutators with the clock variable $q_{c l}$. Therefore $q_{c l}$ cannot possess a sharp value together with either $p$ or $q$ at the time of the photon emission!

As a consequence of Eq. (6) we have the following uncertainty relation ${ }^{4}$ between $p$ and $q_{c l}$ at time $t$

$$
\Delta p \cdot \Delta q_{c l} \geq \frac{t g}{2 c^{2}} \hbar
$$

Since in this case, with $V^{\prime}=0$, it follows from (3) that $p(t)=p(0)-m g t$, we have the following relation between the uncertainties in $p$ and $m$ :

$$
\Delta p=g t \Delta m .
$$

This uncertainty in $m$ must be understood in the following way. As we have just shown, the final measurement result, which may be completely sharp, translates back to a state with width $\Delta p$ at the time of the photon emission. This introduces an uncertainty in the determination of $m$ : different values of $m$ could have led to the same final measurement result because the value of $p(t)$,

\footnotetext{
${ }^{4}$ Analogously to the standard uncertainty relation $\Delta p . \Delta q \geq \frac{1}{2} \hbar$ that follows from the canonical commutation relation $[p, q]=-i \hbar$.
} 
as ascertained from the final measurement is uncertain. The value $\Delta m$ just calculated is the range of $m$-values that is compatible with the width $\Delta p(t)$ and the measurement result. This $\Delta m$ equals the uncertainty with which we can make a prediction about the mass of the photon, on the basis of the measured value $p(0){ }^{5}$

Equation (8), together with the fact that the uncertainty in $q_{c l}$ at $t$ equals the uncertainty $\Delta T$ in the instant of the opening of the shutter and together with $\Delta E=c^{2} \Delta m$, leads to

$$
\Delta E . \Delta T \geq \frac{1}{2} \hbar .
$$

Alternatively, we may focus on $q$ instead of $p$ in order to determine $m$. This would be in line with Bohr's account in which the center of mass of the box is rigidly connected to a pointer that moves along a scale. In this case we find from (3): $\Delta q=\frac{g t^{2}}{2 M} \Delta m$, which together with (7) leads to

$$
\Delta E . \Delta T=c^{2} \Delta m . \Delta q_{c l}=\frac{2 c^{2} M}{g t^{2}} \cdot \Delta q \cdot \Delta q_{c l} \geq \frac{1}{2} \hbar .
$$

So regardless of whether we measure $p$ or $q$, we shall not be able to predict the energy and arrival time of the photon with a smaller latitude than allowed by the time-energy uncertainty relation.

If $V^{\prime}$ depends on $q$, the calculations become more complicated. Let us have a look at the case suggested in Bohr's account, in which the box is suspended from a spring. We can model this situation by assuming a harmonic force $-k q$, with $k$ a positive constant. This leads to the following Heisenberg equations of motion:

$$
\dot{q}(t)=p / M \quad, \quad \dot{p}(t)=-m g-k q,
$$

and therefore

$$
\ddot{q}(t)=-\frac{m}{M} g-\frac{k}{M} q .
$$

The solutions of these equations are given by:

$$
q(t)=\frac{m g}{k}(\cos \omega t-1)+q(0) \cos \omega t+\frac{p(0)}{M \omega} \sin \omega t,
$$

and

$$
p(t)=-\frac{M m \omega g}{k}(\sin \omega t)-M \omega q(0) \sin \omega t+p(0) \cos \omega t,
$$

in which $\omega^{2}=k / M$.

\footnotetext{
${ }^{5}$ Since the state of the box is a mixture of components with different $m$-values, we may argue about the situation in a classical way: different possible values of $m$ lead to uncertainty in the evolution and consequently to an uncertainty in $p$.
} 
For the uncertainties in $m$ connected with $\Delta q$ and $\Delta p$, respectively, we thus find (for $\omega t<<M / m$ ):

$$
\Delta m_{q}(t)=\frac{k}{g(1-\cos \omega t)} \Delta q(t),
$$

and

$$
\Delta m_{p}(t)=\left|\frac{k}{M \omega g \sin \omega t}\right| \Delta p(t) .
$$

The commutators $\left[p, q_{c l}\right]$ and $\left[q, q_{c l}\right]$ in this case, with $V(q)=\frac{1}{2} k q^{2}$, satisfy the equations

$$
\begin{gathered}
\frac{d}{d t}\left[p, q_{c l}\right]=\frac{g}{c^{2}} i \hbar-k\left[q, q_{c l}\right], \\
\frac{d}{d t}\left[q, q_{c l}\right]=\frac{1}{M}\left[p, q_{c l}\right],
\end{gathered}
$$

so that

$$
\frac{d^{2}}{d t^{2}}\left[p, q_{c l}\right]=-\frac{k}{M}\left[p, q_{c l}\right] .
$$

It follows that

$$
\begin{gathered}
{\left[p, q_{c l}\right]=\frac{g}{c^{2}} \frac{\sin \omega t}{\omega} i \hbar} \\
{\left[q, q_{c l}\right]=\frac{g}{c^{2}} \frac{1-\cos \omega t}{M \omega^{2}} i \hbar .}
\end{gathered}
$$

This yields the uncertainty relations

$$
\begin{gathered}
\Delta p . \Delta q_{c l} \geq\left|\frac{g \sin \omega t}{2 \omega c^{2}}\right| \hbar, \\
\Delta q . \Delta q_{c l} \geq \frac{g(1-\cos \omega t)}{2 M \omega^{2} c^{2}} \hbar .
\end{gathered}
$$

Together with equations (16) and (17) this leads to the expected uncertainty relations for $E$ and $T$.

So regardless of whether the box is moving freely or executes a harmonic motion, and regardless of whether we base our predictions on a determination of $q$ or on a determination of $p$, we always find $\Delta E . \Delta T \geq \frac{1}{2} \hbar$. The uncertainties in $q$ and $p$ on the one hand, and in $q_{c l}$ on the other, guarantee that no conflict with the time-energy uncertainty relation for the photon can arise. The essential point is that the box quantities $q_{c l}$ and $m$ (as calculated from either $p$ or $q$ ) form a complementary pair. 


\section{Epilogue}

One might still wonder about the completely general case, with arbitrary $V(q)$. The result of any specific calculation is known beforehand, however: Any spread in the energy (or equivalently mass) of the box, regardless of whether introduced by an uncertainty $\Delta q$ or an uncertainty $\Delta p$, will result in an uncertainty in the evolution of $q_{c l}$ by virtue of the general time-energy relation $[6,2,4]$

$$
\Delta H \cdot \frac{\Delta q_{c l}}{\left\langle q_{c l}\right\rangle} \geq \frac{1}{2} \hbar .
$$

This guarantees in a general way that the uncertainties will come out right. That does not make the above specific calculations irrelevant, however. The latter show in a bottom-up way how the danger of inconsistency is avoided by the dynamics of quantum mechanics. The paradox that $p$ (or $q$ ) and the clock time can be read off simultaneously, and that therefore (seemingly) both $E_{p h}$ and $T_{a r r}$ can be precisely predicted, is dissolved by showing how the validity of complementarity is guaranteed by the details of the dynamics. These calculations put Bohr's historical, qualitative arguments against Einstein on a firm quantitative basis.

Moreover, our calculations exhibit a "fine structure" in the behavior of the uncertainties that does not follow from the general uncertainty relation. As becomes clear from (8), (9), (16), (17), (23) and (24), the uncertainties in $E$ and $T$ are not time independent. Although their products always satisfy the general $E-T$ uncertainty relation, the distribution of uncertainty over the quantities changes in time. This leads to a remarkable conclusion: depending on when the box measurement is performed, the resulting quantum description of the photon differs - in spite of the fact that in the meantime the photon can have reached a distance of lightyears. This is another illustration of the leading idea of the later EPR thought experiment. We may safely assume that pondering the implications of complementarity in the photon-box case has played a pivotal role in Einstein's dissatisfaction with quantum mechanics. At the same time, the experiment nicely illustrates how quantum mechanics consistently deals with this type of correlated systems.

\section{References}

[1] Bohr, N. (1949) 'Discussion with Einstein on Epistemological Problems in Atomic Physics', in Albert Einstein: Philosopher-Scientist, P.A. Schilpp, ed., Open Court, La Salle, 199-241.

[2] Busch, P. (1990) 'On the Energy-Time Uncertainty Relation', Foundations of Physics 20, 1-32; (2007) 'The Time-Energy Uncertainty Relation', arXiv:quant-ph/0105049v3. 
[3] Dieks, D. (1999) 'The Bohr-Einstein Photon Box Debate', in Language, Quantum, Music, M.L. Dalla Chiara et al. eds., Kluwer Academic Publishers, Dordrecht, 283-292.

[4] Hilgevoord, J. (1998) 'The Uncertainty Principle for Energy and Time. II', American Journal of Physics 66, 396-402.

[5] Howard, D. (1990) 'Nicht Sein Kann Was Nicht Sein Darf' in Sixty-Two Years of Uncertainty, A.I. Miller, ed., Plenum, New York, 61-111.

[6] Messiah, A. (1961) Quantum mechanics, Vol. I, North-Holland Publishing Company, Amsterdam, 319-320.

[7] Unruh, W.G. and Opat, G.I. (1979) 'The Bohr-Einstein "Weighing of Energy" Debate', American Journal of Physics 47, 743-744. 